Pacific Journal of Mathematic 


\title{
APPROXIMATIONS WITH LEAST MAXIMUM ERROR
}

\author{
R. G. SELFRIDGE
}

1. Introduction. This paper is concerned with the problem of finding best approximations to a given function, where 'best' is defined in a particular fashion, and the approximation is taken from a given class of functions. The approximated function need only be bounded and single-valued; the range of the independent variable can be any closed interval, where the infinite interval is closed at infinity.

This problem was originally investigated from the point of view of obtaining approximations to be used on automatic computing machines in place of functions with slowly converging Taylor series. The result is also of value in determining formulae for empirically determined functions.

This problem has been studied by many people. The present paper extends previous results, and gives an indication of how these approximations can be obtained. Unfortunately, only a sufficient condition has been given, and the convergence of the iteration method given has not been proved.

2. Theorem. Let us suppose that a function $f(x)$ is to be approximated over the closed interval $[a, b]$, and that $m \leq f(x) \leq M$ in that interval; $f(x)$ need not be continuous but must be single-valued in $[a, b]$. In practice $f(x)$ will usually have a Taylor series. Take $G$ to be a class of functions, called the class of approximating functions, from which an element $k(x)$ is to be chosen to approximate $f(x)$. From here on it is to be understood that all results will be relative to the interval $[a, b]$ and the particular class $G$.

DEFINITION 1. If $h(x)$ is continuous and $h(x) \neq 0$, define

$$
E_{k}=\max _{a \leq x \leq b} \frac{|f(x)-k(x)|}{|h(x)|}
$$

for all $k(x)$ in $G$.

In practice, $h(x)$ will be equal to 1 or equal to $f(x)$, to yield actual error or relative error, between $f(x)$ and $k(x)$. 
DEFINITION 2. The function $g(x) \in G$ is a best approximation to $f(x)$, relative to $h(x)$, if $E_{g} \leq E_{k}$ for all $k(x)$ in $G$.

Definition 3. Set

$$
e(x)=\frac{f(x)-g(x)}{h(x)}
$$

The extrema of $e(x)$ are the relative maxima and minima of $e(x)$. The absolute extrema are the $e_{j}=e\left(x_{j}\right)$ for which $|e(x)| \leq\left|e_{j}\right|$. It should be pointed out that $d e / d x$ need not be zero for extrema at the points $a, b$ of the closed interval $[a, b]$.

Now consider $G$ to be the class of functions of the form

$$
Q\left[K(x) \sum_{i=0}^{n} a_{i} x^{p_{i}}\right]
$$

for some fixed $n$, where $Q(y), K(y)$ are fixed functions, and $p_{i}(i=0,1, \cdots, n)$ is a sequence of positive integers such that $0=p_{0}<p_{1}<\cdots<p_{n}$. It is assumed that $Q(y)$ has an inverse $P(y)$ with a continuous first derivative $P^{\prime}(y)$, and that $P^{\prime}(y) \neq 0$ in the interval $\left[m-H E_{g}, M+H E_{g}\right]$, where $H$ is the maximum absolute value of $h(x)$ as used in Definition 1.

Thus $G$ is a class of functions dependent on the $n+1$ coefficients $a_{i}$, and is, in a sense, a class of generalized polynomials. This choice of $G$ will cover many of the practical cases of approximation with the exception of rational approximations with free coefficients in both numerator and denominator. The requirements of unisolvence used in the approximations of Motzkin [2] include the condition that the difference of two approximations have $n$ or fewer roots in $[a, b]$. This restriction and similar ones that lead to Tchebycheff or Descartes approximations, as used by Bernstein [1], will not permit such examples as powers of polynomials, or polynomials of degree greater than $n$ if $[a, b]$ includes the origin, or products of polynomials and functions that have roots in $[a, b]$. The simplest case of approximation by a polynomial of degree $n$ has been handled by many methods, including the foregoing and others, such as that of de la Vallee Poussin [3]. The results of Motzkin [2] will handle many rational approximations that this paper cannot, and furthermore they supply a necessary and sufficient condition.

LEMMA. If $0 \leq x_{0}<x_{1}<\cdots<x_{n+1}$, then 


$$
A_{k}^{n}(x) \equiv A\left(x_{0}, x_{1}, \cdots, x_{k-1}, x_{k+1}, \cdots, x_{n+1}\right) \equiv\left|\begin{array}{cccc}
1 & x_{0}^{p_{1}} & \cdots & x_{0}^{p_{n}} \\
1 & x_{1}^{p_{1}} & \cdots & x_{1}^{p_{n}} \\
\cdots & \cdots & \cdots \\
1 & x_{k-1}^{p_{1}} & \cdots & x_{k-1}^{p_{n}} \\
1 & x_{k+1}^{p_{1}} & \cdots & x_{k+1}^{p_{n}} \\
& \cdots & \cdots & x_{k+1}^{p_{n}} \\
1 & x_{n+1}^{p_{1}} & \cdots & x_{n+1}^{p_{n+1}}
\end{array}\right|
$$

is positive definite.

Proof. Clearly this is true for $n=1$. Suppose it is true for all $m<n$. Then expand $A_{k}^{n}(x)$ in terms of $x_{0}$. By the induction hypothesis, every $n$ by $n$ minor is positive. Thus

$$
A_{k}^{n}(x)=B\left(x_{0}\right)=B_{0}-B_{1} x_{0}^{p_{1}}+\cdots+(-1)^{n} B_{n} x_{0}^{p_{n}},
$$

where each $B_{i}>0$. By Descartes' rule of signs, the equation $B\left(x_{0}\right)=0$ has $n$ positive roots, namely $x_{0}=x_{i}$ for $i=1,2, \cdots, k-1, k+1, \cdots, n+1$. Also, by the induction hypothesis, $B_{0}$ is positive; so we have

$$
A_{k}^{n}(x)=R(x) \prod_{i \neq k}\left(x_{i}-x_{0}\right)
$$

where $R(x)$ is positive. Since $x_{0}<x_{i}, A_{k}^{n}(x)$ is positive. The induction holds and the lemma is proved.

THEOREM. A function $g(x)$ in $G$, where $G$ is as above, is a best approximation to $f(x)$ in the closed interval $[a, b]$ if the following conditions are satisfied:

1) The function $e(x)$ has $n+2$ absolute extrema $e_{j}=e\left(x_{j}\right)$ such that $x_{j}<x_{j+1}$, and $e_{j}=t_{j} e_{0}$, with $t_{j}= \pm 1$.

2) The values $(-1)^{j} t_{j} h\left(x_{j}\right) A_{j}^{n} / K\left(x_{j}\right)$ are all nonzero, and are all positive or all negative.

Proof. Consider the $n+2$ points $x_{j}$, where $e_{j}=e\left(x_{j}\right)$ is an absolute extremum. Let us assume that $e_{0}$ is positive. Take any other approximation that is as good as or better than $g(x)$; that is, $E_{k} \leq E_{g}$. Set 


$$
e^{\prime}=\frac{f(x)-k(x)}{h(x)}
$$

and let $e_{j}^{\prime}=e^{\prime}\left(x_{j}\right)$. Then since $E_{k} \leq E_{g}$ one must have $e_{j}^{\prime}=\theta_{j} e_{j}$, where $\left|\theta_{j}\right| \leq 1$

Consider now the $n+2$ equations in the $n+1$ unknowns $a_{i}$,

(1) $f\left(x_{j}\right)-Q\left[K\left(x_{j}\right) \sum_{i=0}^{n} a_{i} x_{j}^{p_{i}}\right]=h\left(x_{j}\right) e\left(x_{j}\right) \quad(j=0,1, \cdots, n+1)$.

Rewritten in simpler notation, (1) is

$$
f_{j}-Q\left(K_{j} \sum_{i=0}^{n} a_{i} x_{j}^{p_{i}}\right)=h_{j} e_{j}
$$

or

$$
\sum_{i=0}^{n} a_{i} x_{j}^{p_{i}}=P\left(f_{j}-h_{j} e_{j}\right) / K_{j} \quad(j=0,1, \cdots, n+1) .
$$

This is possible since $Q$ is assumed to have an inverse; and by hypothesis $K_{j} \neq 0$.

For a given set of $e_{j}$ 's there is a solution to (2) if and only if the determinant of the coefficients is zero:

(3)

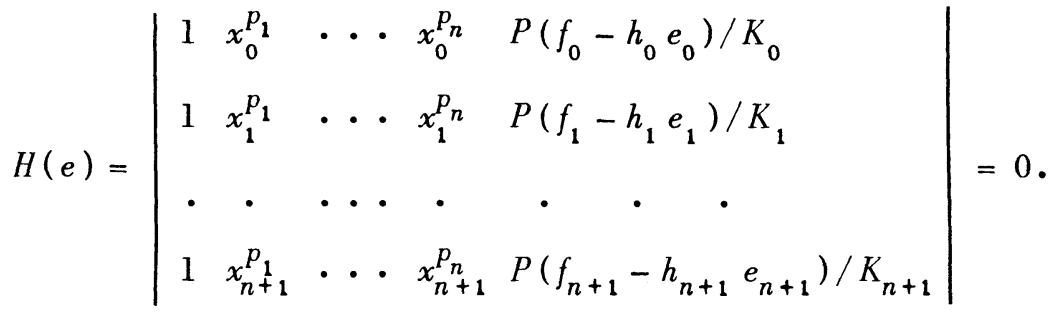

Also, since by hypothesis $A_{j}^{n} \neq 0$, if $H(e)=0$ there is a unique solution. The two sets $e_{j}$ and $e_{j}^{\prime}$ must both satisfy $(3)$; that is, $H(e)=H\left(e^{\prime}\right)=0$. Now set

$$
e_{j}-e_{j}^{\prime}=\delta_{j}=e_{j}\left(1-\theta_{j}\right)
$$

By the mean-value theorem for several variables, we have 


$$
H(e)-H\left(e^{\prime}\right)=\sum_{j=0}^{n+1}\left(e_{j}-e_{j}^{\prime}\right) \frac{\partial H}{\partial e_{j}},
$$

where each of the partial derivatives is evaluated at some intermediate point; (4) may be written as

$$
H(e)-H\left(e^{\prime}\right)=0=\sum_{j=0}^{n+1} \delta_{j} \frac{\partial H}{\partial e_{j}} .
$$

The partial derivative $\partial H / \partial e_{j}$ is given by

$$
\frac{\partial H}{\partial e_{j}}=\left|\begin{array}{ccccc}
1 & x_{0}^{p_{1}} & \cdots & x_{0}^{p_{n}} & P_{0} / K_{0} \\
\cdots & \cdot & \cdots & \cdots & \cdots \\
1 & x_{j-1}^{p_{1}} & \cdots & x_{j-1}^{p_{n}} & P_{j-1} / K_{j-1} \\
0 & 0 & \cdots & 0 & -h_{j} P_{j}^{\prime} / K_{j} \\
1 & x_{j+1}^{p_{1}} & \cdots & x_{j+1}^{p_{n}} & P_{j+1} / K_{j+1} \\
\cdot & \cdot & \cdots & \cdot & \cdots \\
1 & x_{n+1}^{p_{1}} & \cdots & x_{n+1}^{p_{n}} & P_{n+1} / K_{n+1}
\end{array}\right|,
$$

where $P_{i}$ and $P_{j}^{\prime}$ are evaluated at some intermediate point.

This may be written, by expansion on the $j$ th row, in the form

$$
\frac{\partial H}{\partial e_{j}}=(-1)^{n+j+1} P^{\prime}\left(f_{j}-h_{j} e_{j}+h_{j} \phi_{j} \delta_{j}\right) h_{j} A_{j}^{n} / K_{j},
$$

where $0 \leq \phi_{i} \leq 1$

Thus $\partial H / \partial e_{j}$ is dependent only on the different $x_{i}$, and on $h_{j}, f_{j}$; it is not dependent on $e_{i}, i \neq j$.

Now we have

$$
-h_{j} e_{j}+h_{j} \phi_{j} \delta_{j}=-h_{j} e_{j}\left[1-\phi_{j}\left(1-\theta_{j}\right)\right]=-h_{j} e_{j} \zeta_{j},
$$

where $\left|\zeta_{j}\right| \leq 1$ since $0 \leq \phi_{j} \leq 1$ and $\left|\theta_{j}\right| \leq 1$.

Therefore 


$$
P^{\prime}\left(f_{j}-h_{j} e_{j}+h_{j} \phi_{j} \delta_{j}\right)=P^{\prime}\left(f_{j}-h_{j} e_{j} \zeta_{j}\right)
$$

and we have

$$
\frac{\partial H}{\partial e_{j}}=(-1)^{n+j+1} P^{\prime}\left(f_{j}-h_{j} e_{j} \zeta_{j}\right) h_{j} A_{j}^{n} / K_{j}
$$

or, rewriting ( 5 ),

$$
\sum_{j=0}^{n+1} \delta_{j} \frac{\partial H}{\partial e_{j}}=\sum_{j=0}^{n+1}(-1)^{n+j+1} P^{\prime}\left(f_{j}-h_{j} e_{j} \zeta_{j}\right) h_{j} A_{j}^{n} \delta_{j} / K_{j}=0
$$

Now by hypothesis $e_{j}=t_{j} e_{0}$, and we set $\delta_{j}=e_{j}\left(1-\theta_{j}\right)$. Then (8) becomes

$$
\sum_{j=0}^{n+1}(-1)^{j} P^{\prime}\left(f_{j}-h_{j} e_{j} \zeta_{j}\right) h_{j} A_{j}^{n} e_{0} t_{j}\left(1-\theta_{j}\right) / K_{j}=0
$$

Now $P^{\prime}\left(f_{j}-h_{j} e_{j} \zeta_{j}\right)$ is always positive, or always negative, by assumption on $Q(x)$; hence, by the second condition of the hypothesis, (9) may be written as

$$
\sum_{j=0}^{n+1} e_{0} T_{j}\left(1-\theta_{j}\right)=0
$$

where $T_{j}$ is always positive.

Equation (10) can be satisfied only if $e_{0}=0$, in which case $f(x)=g(x)$, or $\theta_{j}=1$; then $k(x)=g(x)$ at the points $x_{j}$, and thus $k(x)=g(x)$ for $x$ in $[a, b]$.

Therefore $g(x)$ is a best approximation, and is unique.

COROLLARY 1. If the origin is not contained in $[a, b]$ and $K(x)$ and $h(x)$ do not change sign in $[a, b]$, then, by Lemma $1, g(x)$ is a best approximation if $e(x)$ has $n+2$ absolute extrema such that $e_{j}=e_{0}(-1)^{j}$ and $x_{j}<x_{j+1}$.

COROLLARY 2. If not all $A_{j}^{n} \neq 0$, then $g(x)$ is still a best approximation provided that at least one $A_{j}^{n} \neq 0$; but $g(x)$ is not necessarily unique.

This follows since (9) still holds; and (10) still holds but with $T_{j} \geq 0$ and at least one $T_{j} \neq 0$. Thus $k(x)$ cannot be better than $g(x)$, but need not be 
identical with $g(k)$.

COROLLARY 3. If $P^{\prime}(y)$ does change sign in

$$
m-H E_{g} \leq y \leq M+H E_{g},
$$

then the theorem still holds, but with condition 2 ) replaced by: for

$\left.2^{\prime}\right)$ The value $(-1)^{j} t_{j} h_{j} A_{j}^{n} P^{\prime}\left(y_{j}\right) / K_{j}$ is nonzero and positive (negative)

$$
f_{j}-\left|h_{j} e_{0}\right| \leq y_{j} \leq\left|h_{j} e_{0}\right|+f_{j} \quad(j=0,1, \cdots, n+1) .
$$

This follows from (9) since it is sufficient that $P^{\prime}\left(f_{j}-h_{j} e_{j} \zeta_{j}\right)$ not change sign for any $\zeta_{j}$.

3. Approximations. In practice the class $G$ usually has simple functions $Q(x), K(x)$. The function $K(x)$ is chosen to remove some awkward point of $f(x)$, such as a point with an infinite derivative, as occurs for example in $\sin ^{-1} x$, while the function $Q(x)$ is taken to be a function like $x, x^{2}$ (this requires care, because there is no unique inverse), or $1 / x$. The requirements of the theorem force the approximations to be continuous if $K(x)$ is continuous.

The procedure for finding $g(x)$ is to guess some initial value of $e_{0}$, to guess a set of points $x_{i}$, and to set up $n+1$ equations in order to find the approximation that will go through the $n+1$ points $x_{i}, f_{i}-t_{i} e_{0}$. This requires using $\mathrm{n}+2$ points to compute the $t_{i}$. If the error curve is now plotted, then $e(x)$ will have $n+2$ absolute extrema with values $\mathrm{e}_{j}^{\prime}$ at the points $x_{j}^{\prime}$, and the new values will not differ too greatly from the original $x_{i}$. That is, $e_{j}^{\prime} \approx t_{j} e_{0}$ and $x_{j} \approx x_{j}^{\prime}$. Now by averaging, or solution of (3), we find a new $e_{0}$ for the points $x_{j}^{\prime}$, and repeat the process. This process will usually converge to the desired $g(x)$, but a criterion for convergence has not yet been found. Experience has shown that if the origin is not in $[a, b]$, then convergence is rapid, even from a poor first approximation, but that if the origin is in $[a, b]$ it will be necessary to solve ( 3 ) on each iteration at first, and even then the iteration may not converge for a poor first approximation.

Further difficulty can be experienced in that, if there are more than $n+2$ absolute extrema, one choice of $n+2$ may not show that an approximation is best, while another choice will show that the approximation is best. It is therefore necessary to apply the test to all possible sets of $n+2$ absolute extrema to show that a given case is best. 
As an example of the iteration process, consider the problem of approximating $\sin x$ in the range $[0,1]$ by a form $a x+b x^{3}$. This form satisfies the conditions of Corollary 1 , so that the iteration is fairly simple. The functions are considered here in $x$-intervals of length .02 , and the computations have been rounded off. Suppose that at some intermediate stage one has:

$$
a=.99987, b=-.159403 \text {. }
$$

This yields an error curve with the extrema

$$
e_{1}=.000006, e_{2}=-.001, e_{3}=.001
$$

at the points

$$
x_{1}=.08, x_{2}=.76, x_{3}=1.0 \text {. }
$$

By averaging, or a solution of (3), one finds a new $e_{0}^{\prime}=.0007$, and one now finds a new approximation with an error curve that goes through the points

$$
(.76,-.0007),(1.0, .0007)
$$

By a solution of (3), the new error curve could also be made to go through the correct value for $x_{1}$, but the extra computation to get this value is frequently not worth the effort. The new curve has

$$
a=.99850, b=-.157731 \text {, }
$$

with extrema at the points

$$
(.24, .00024),(.76,-.0007),(1.0, .0007)
$$

Averaging these extrema yields $e_{0}=.0005$, and the approximation with error curve going through the points

$$
(.76,-.0005),(1.0, .0005)
$$

has

$$
a=.997605, b=-.156634,
$$

and extrema at the points

$$
(.32, .00046),(.8,-.00053),(1.0, .0005)
$$

Repetition of this process finally stabilizes with the absolute extrema at 
$.3, .8,1.0$, and we have

$$
\sin x \approx .997491 x-.1565191 x^{3}
$$

with the maximum error less than $5 \times 10^{-4}$.

Further refinement is of course possible, by carrying more places in the computation, or by taking more points in $[0,1]$; but from the point of view of computational use the additional work is not justified.

The choice of form to be used in an approximation can be decided at present only by trial, as is also the case in all the different ways of short-cutting the iteration.

\section{REFERENCES}

1. S. Bernstein, Lecons sur les propriétés extrémales, et la meilleure approximation des fonctions analytiques d'une variable réelle, Gauthier-Villars, Paris, 1926.

2. Th. Motzkin, Approximation by curves of a unisolvent family, Bull. Amer. Math. Soc. 55 (1949), $789-793$.

3. Ch. J. de la Vallée Poussin, Sur les polynomes d'approximation et la représentation approchée d'un angle, Acad. Roy. Belgique. Bull. Cl. Sci. (1910), 808-844.

Naval Ordnance Test Station

China Lake, California 



\section{PACIFIC JOURNAL OF MATHEMATICS}

\section{EDITORS}

\author{
R. M. Foeinson \\ University of California \\ Berkeley 4, California \\ E. HEWITt \\ University of Washington \\ Seattle 5, Washington
}

R. P. DILWOR TH

California Institute of Technology

Pasadena 4, California

E. F. BECKENBACH

University of California

Los Angeles 24, California

\section{ASSOCIATE EDITORS}

$\begin{array}{llll}\text { H. BUSEMANN } & \text { P. R. HALMOS } & \text { BøRGE JESSEN } & \text { J. J. STOKER } \\ \text { HERBERT FFDERER } & \text { HEINZ HOPF } & \text { PAUL LÉVY } & \text { E. G. STR AUS } \\ \text { MARSHALL IIALI } & \text { R. D. JAMES } & \text { GEORGE PÓLYA } & \text { KỎSAKU YOSIDA }\end{array}$

\section{SPONSORS}

UNIVERSITY OF BRITISH COLUMBIA CAIIFORNIA INSTITUTE OF TECHNOLOGY UNIVERSITY OF CALIFORNIA, BERKELEY UNIVERSITY OF CAILIFORNIA, DAVIS UNIVERSITY OF CALIFORNIA, LOS ANGELES UNIVERSITY OF CALIFORNIA, SANTA BARBARA UNIVERSITY OF NEVADA OREGON STATE COLLEGE UNIVERSITY OF OREGON
UNIVERSITY OF SOUTHERN CALIFORNIA STANFORD RESEARCH INSTITUTE STANFORD UNIVERSITY WASHINGTON STATE COLLEGE UNIVERSITY OF WASHINGTON

AMERICAN MATHEMATICAL SOCIETY NATIONAL BUREAU OF STANDARDS, INSTITUTE FOR NUMERICAL ANALYSIS

$$
\begin{gathered}
\text { Vari-Type Composition by } \\
\text { Elaine Barth } \\
\text { Delores Wierman } \\
\text { With the cooperation of } \\
\text { E. F. Beckenbach } \\
\text { E. G. Straus }
\end{gathered}
$$

Printed in the United States of America by Edwards Brothers, Inc., Ann Arbor, Michigan 


\section{Pacific Journal of Mathematics}

\section{Vol. 3, No. 1 \\ March, 1953}

Herbert Busemann, Volume in terms of concurrent cross-sections ......... 1

L. Carlitz, Some special equations in a finite field ................. 13

Homer V. Craig and Billie Braden Townsend, On certain metric

extensors ....................................... 25

Philip J. Davis and Henry Pollak, Linear functionals and analytic

continuation problems ............................. 47

Jacob C. E. Dekker, The constructivity of maximal dual ideals in certain

Boolean algebras ................................. 73

Harley M. Flanders, The norm function of an algebraic field extension .... 103

Marshall Hall, Subgroups of free products . . . . . . . . . . . . . . . . . 115

Israel (Yitzchak) Nathan Herstein, Finite multiplicative subgroups in

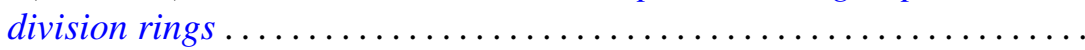

Joseph Lawson Hodges, Jr. and Murray Rosenblatt, Recurrence-time moments in random walks ............................ 127

Alfred Horn, The normal completion of a subset of a complete lattice and lattices of continuous functions ........................ 137

Fulton Koehler, Estimates for the errors in the Rayleigh-Ritz method...... 153

M. H. Martin, The Monge-Ampère partial differential equation

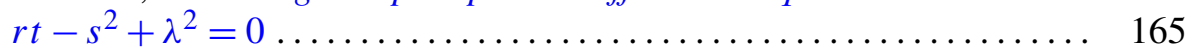

John E. Maxfield, Normal k-tuples ......................... 189

Jack E. McLaughlin, Structured theorems for relatively complemented lattices............................................ 197

William H. Mills, A system of quadratic Diophantine equations ......... 209

T. S. Motzkin, Ernst Gabor Straus and F. A. Valentine, The number of farthest points ................................. 221

G. Power, Forces on the boundary of a dielectric ............... 233

Ralph Gordon Selfridge, Approximations with least maximum error....... 247 